(C) 2006 International Press

Adv. Theor. Math. Phys. 10 (2006) 591-602

\title{
Seidel's mirror map for abelian varieties
}

\author{
Marco Aldi and Eric Zaslow \\ Department of Mathematics, Northwestern University, \\ 2033, Sheridan Road, Evanston, IL 60208, USA \\ m.aldi@math.northwestern.edu; zaslow@math.northwestern.edu
}

\begin{abstract}
We compute Seidel's mirror map for abelian varieties by constructing the homogeneous coordinate rings from the Fukaya category of the symplectic mirrors. The computations are feasible, as only linear holomorphic disks contribute to the Fukaya composition in the case of the planar Lagrangians used. The map depends on a symplectomorphism $\rho$ representing the large complex structure monodromy. For the example of the two-torus, different families of elliptic curves are obtained by using different $\rho$ 's which are linear in the universal cover. In the case where $\rho$ is merely affine linear in the universal cover, the commutative elliptic curve mirror is embedded in noncommutative projective space. The case of Kummer surfaces is also considered.
\end{abstract}

\section{Introduction}

In [14], we constructed Seidel's mirror map for the two-torus. Starting from the Fukaya category of a symplectic two-torus $X$, we computed the homogeneous coordinate ring $\mathcal{R}$ of the mirror elliptic curve $Y$. The proof of Kontsevich's conjecture for the elliptic curve [12] allowed us to do so. Namely, $D F u k(X) \cong D(Y)$ implies that $\mathcal{R}$ is computable on $X$ alone, i.e.,

e-print archive: http://lanl.arXiv.org/abs/math.SG/0512229a 
we have

$$
\mathcal{R}=\bigoplus_{k=0}^{\infty} \Gamma\left(\mathcal{O}_{Y}(k)\right)=\bigoplus_{k=0}^{\infty} \operatorname{Hom}_{D \operatorname{Fuk}(X)}(\psi(\mathcal{O}), \psi(\mathcal{O}(k)))
$$

where $\psi$ is the equivalence of categories (see also [1]). In fact, in the above, $\mathcal{O}$ can be replaced by any line bundle $\mathcal{L}$. As the mirror of the automorphism $-\otimes \mathcal{O}_{Y}(1)$ is known to be the symplectomorphism $\rho$ effecting the monodromy around the large complex structure limit, the right-hand side can be computed entirely from the Fukaya category, once we choose any Fukaya object mirror to some line bundle. In this paper, we extend the computation of [14] to higher-dimensional abelian varieties. These cases were treated in [7] and [10], where partial results were obtained toward Kontsevich's conjecture. Those results imply the existence of some of the findings in this paper, although our methods are more direct. We find that the homogeneous coordinate ring of the mirror abelian variety is described by the computation on the right-hand side of equation (1.1). Specifically, we find that the theta relations of the mirror abelian variety are obeyed by the intersection points mirror to the theta functions. Computations are made feasible by the fact that the objects in the Fukaya category are all linear planes in the universal cover.

In addition, we explore the dependence of the homogeneous coordinate ring on $\rho$. Specifically, different choices for $\rho$ yield different families of abelian varieties. When $\rho(x, y)$ is not strictly linear but contains a translation, the mirror elliptic curve sits as a commutative variety inside a noncommutative projective space, not unlike the situation found in [3]. The Hesse family of elliptic curves was found to be dual to $\rho(x, y)=(x, y+3 x)$, in [14]. If we choose $\rho(x, y)=(x, y+x)$, we find the universal family of elliptic curves inside weighted projective space $\mathbb{P}(1,2,3)$ corresponding to a quasihomogeneous coordinate ring with corresponding weights. In the four-dimensional case, $\mathbb{Z} / 2 \mathbb{Z}$ invariant maps on the four torus provide a mirror map for Kummer K3 surfaces.

\section{Definitions}

Our main objects of study are symplectic tori with B-field admitting the structure of Lagrangian brane fibration with section (see $[9,11])$. This motivates the following definitions. Let $V$ be a real vector space of dimension $2 n$ together with a complexified symplectic form $i \omega+b$ and a basis 
$l_{1} \ldots, l_{n}, l_{1}^{\prime}, \ldots, l_{n}^{\prime}$ for which $(i \omega+b)\left(l_{i}^{\prime}, l_{j}\right)=i M_{i j}+B_{i j}$ or, in matrix form,

$$
\omega=\left(\begin{array}{cc}
0 & -M^{\mathrm{T}} \\
M & 0
\end{array}\right) \quad b=\left(\begin{array}{cc}
0 & -B^{\mathrm{T}} \\
B & 0
\end{array}\right)
$$

where $M=\left(M_{i j}\right)$ and $B=\left(B_{i j}\right)$ are invertible $n \times n$ matrices. Moreover, let

$$
\Lambda_{0}:=\mathbb{Z}\left\langle l_{1}, \ldots, l_{n}\right\rangle, \quad \Lambda^{\prime}:=\mathbb{Z}\left\langle l_{1}^{\prime}, \ldots, l_{n}^{\prime}\right\rangle .
$$

Consider the Lagrangian subspaces $L_{0}:=\Lambda_{0} \otimes \mathbb{R}, L^{\prime}:=\Lambda^{\prime} \otimes \mathbb{R}$. Suppose that there is a lattice homomorphism $f: \Lambda_{0} \rightarrow \Lambda^{\prime}$ such that its $\mathbb{R}$-linear extension (also denoted by $f$ ) satisfies $f\left(l_{i}\right)=\sum_{j} N_{j i} l_{j}^{\prime}$ and let $N:=\left(N_{i j}\right)$. Then the following are equivalent

(1) The graph $L_{f}:=\left\{l+f(l) \mid l \in L_{0}\right\}$ is Lagrangian

(2) $N^{\mathrm{T}} M$ and $N^{\mathrm{T}} B$ are symmetric

(3) $i \omega+b$ admits the compatible complex structure

$$
J_{f}:=\left(\begin{array}{cc}
0 & -N^{-1} \\
N & 0
\end{array}\right)
$$

(4) $i \omega+b$ admits the symplectomorphism

$$
\rho_{f}:=\left(\begin{array}{cc}
\mathrm{Id} & 0 \\
N & \mathrm{Id}
\end{array}\right) .
$$

Notice that if $f$ satisfies these equivalent conditions, then so does $k f$ for all $k \neq 0$ and $\rho_{(k f)}=\rho_{f}^{k}$. In more general settings, we drop the subscript of $\rho$.

Consider now the quotient torus $T:=V /\left(\Lambda_{0} \oplus \Lambda^{\prime}\right)$. Each $L_{k}:=L_{k f}=$ $\rho_{f}^{k} L_{0}$ has rational slope with respect to the lattice and so it descends to a Lagrangian subtorus, also denoted by $L_{k}$. We are interested in the (derived) Fukaya subcategory generated by these Lagrangian subtori. Define

$$
\Lambda\left(L_{k}\right)_{0}:=f^{-1}\left(\frac{1}{k} \Lambda^{\prime}\right), \quad K\left(L_{k}\right)_{0}:=\frac{\Lambda\left(L_{k}\right)_{0}}{\Lambda_{0}} .
$$

Then for $k_{1} \neq k_{2}$, we have the following isomorphisms of vector spaces

$$
\begin{aligned}
\operatorname{Hom}\left(L_{k_{1}}, L_{k_{2}}\right) & \cong\left\langle L_{k_{1}} \cap L_{k_{2}}\right\rangle \cong\left\langle\left\{\left(l, l^{\prime}\right) \mid l^{\prime}=k_{1} f(l)=k_{2} f(l) \bmod \Lambda^{\prime}\right\}\right\rangle \\
& \cong\left\langle K\left(L_{k_{2}-k_{1}}\right)_{0}\right\rangle \cong\left\langle L_{0} \cap L_{k_{2}-k_{1}}\right\rangle \cong \operatorname{Hom}\left(L_{0}, L_{k_{2}-k_{1}}\right) .
\end{aligned}
$$

Since $\rho_{f}^{k_{2}-k_{1}}$ is a symplectomorphism, the first term and last one are isomorphic as whatever structure the Fukaya category is enriched over. Using these isomorphisms, the composition

$$
m: \operatorname{Hom}\left(L_{0}, L_{k_{1}}\right) \otimes \operatorname{Hom}\left(L_{0}, L_{k_{2}}\right) \longrightarrow \operatorname{Hom}\left(L_{0}, L_{k_{1}+k_{2}}\right)
$$

is well defined. 


\section{Only planar disks contribute}

The results in this section are not new. See for example [7].

All compositions relevant to our computation involve calculating holomorphic maps from a disk with three marked points on the boundary. The three intervals on the boundary between pairs of marked points must be mapped to three Lagrangians, with the points sent to intersections of those Lagrangians. In our case, the three Lagrangians are all related to the base of the Lagrangian fibration $L_{0}$ by the symplectomorphism $\rho_{f}$ linear in the periodic coordinates, thus they all lift to planes in the universal cover $V$.

Since the image of a holomorphic map from a disk is connected, in the universal cover, only one preimage of a Lagrangian will be relevant to any given disk. As a result, we may perform our calculation in the universal cover. Further, we may define coordinates such that the preimages of $L_{0}$ and $L_{k_{1}}$ intersect at the origin $Y_{0}=0 \in V$. Let $Y_{1}$ be the second vertex, where $Y_{1} \in L_{0} \cap L_{k_{2}-k_{1}}$. Then the minimal choice for the third vertex is $Y_{2}=\left(\left(k_{2}-k_{1}\right) / k_{2}\right) Y_{1} \in L_{0} \cap L_{k_{2}}$. With respect to the complex structure $J_{f}$, the holomorphic map $g: D \rightarrow V$ may be constructed as

$$
z_{m}(y)=a_{m} y \quad m \geq 1
$$

where $a_{m}$ are the coefficients in the decomposition $Y_{1}=\sum_{m=1}^{n} a_{m} l_{m}$, and $y=y_{1}+i y_{2}$ is the holomorphic coordinate on a triangular domain (disk) $D$ in the complex plane defined by the vertices 0,1 and $\left(k_{2} /\left(k_{2}-k_{1}\right)\right)\left(1+k_{1} i\right)$. The three legs of this triangle have slope $0, k_{1}$ and $k_{2}$, and we call these boundary intervals $C_{0}, C_{1}$ and $C_{2}$, respectively. The map $g$ is easily shown to satisfy all boundary conditions. Since the universal cover $V$ is a vector space, any other holomorphic map $g^{\prime}$ satisfying the boundary conditions may be expressed as $g^{\prime}=g+\xi$, with $\xi$ a vector-valued holomorphic function. Further, since $g$ and $g^{\prime}$ satisfy the same affine linear boundary conditions, the boundary conditions on $\xi$ are strictly linear. Explicitly, we have

$$
C_{0}: \bar{\xi}_{j}=\xi_{j} ; \quad C_{1}: \bar{\xi}_{j}=\mu_{\alpha\left(k_{1}\right)} \xi_{j} ; \quad \bar{\xi}_{j}=\mu_{\alpha\left(k_{2}\right)} \xi_{j}, \quad j=1,2,
$$

where we have defined $\alpha(n)=1+n i$ and $\mu_{\alpha}=\bar{\alpha} / \alpha$. Note that $\left|\mu_{\alpha}\right|=1$. Consider $\xi \equiv \xi_{j}$, where $j$ could be 1 or 2 . Since $\xi$ is homolorphic, it has a convergent power series expansion $\xi=\sum_{n} c_{n} y^{n}$, and the boundary condition on $C_{0}$ requires that the $c_{n}$ are real. For the boundary $C_{1}$, put $\alpha \equiv \alpha\left(k_{1}\right)=$ $1+k_{1} i$ and note that on $C_{1}$ we may write $y=s \alpha$, where $s$ is real and runs from 0 to $k_{2} /\left(k_{2}-k_{1}\right)$. Then we write the boundary conditions as

$$
\left.\bar{\xi}\right|_{C_{1}}=\sum_{n=0}^{\infty} c_{n}(s \bar{\alpha})^{n}=\left.\mu_{\alpha} \xi\right|_{C_{d}}=\left(\frac{\bar{\alpha}}{\alpha}\right) \sum_{n=0}^{\infty} c_{n}(s \alpha)^{n} .
$$


Equating powers of $s$ requires that for all $n$ such that $C_{n}$ is nonzero, we have $\left(\mu_{\alpha}\right)^{n-1}=1$. If $n \neq 1$ we learn that $\mu_{\alpha}$ must be a root of unity. However, $\mu_{\alpha}=-\left(\left(k_{1}^{2}-1\right) /\left(k_{1}^{1}+1\right)\right)-\left(2 k_{1} /\left(k_{1}^{2}+1\right)\right) i=q_{1}+q_{2} i$, with $q_{1}$ and $q_{2}$ rational. But only the first, second and fourth roots of unity belong to $\mathbb{Q}(i)$, and $\mu_{\alpha}$ is none of these. Finally, the case $n=1$ may be considered separately and shown not to satisfy the boundary condition along $C_{2}$. Thus all $c_{n}=0$ and $\xi \equiv 0$. This completes the proof that no non-planar holomorphic disks contribute to the Fukaya product of Lagrangian planes.

\section{Relations}

In the previous section, we found that only planar disks contribute to the product (2.1). In this section, we use this information to derive an explicit expression for the product and some results about the structure of the $\operatorname{ring} \mathcal{R}$.

The basic holomorphic disk with vertices $Y_{0} \in L_{0} \cap L_{k_{1}}, \rho^{k_{1}}\left(Y_{1}\right) \in \rho^{k_{1}}\left(L_{0} \cap\right.$ $\left.L_{k_{2}}\right)$ and $Y_{2} \in L_{0} \cap L_{k_{1}+k_{2}}$ has symplectic area $\left(k_{1} k_{2} /\left(2\left(k_{1}+k_{2}\right)\right)\right) \omega\left(Y_{1}-\right.$ $\left.Y_{0}, f\left(Y_{1}-Y_{0}\right)\right)$. The other triangles relevant to the same composition are taken into account by the generating function

$$
A_{Y_{1}-Y_{0}}^{[k]}=\sum_{\lambda \in \Lambda_{0}} \mathrm{e}^{-\pi k(\omega-i b)\left(Y_{1}-Y_{0}-\lambda, f\left(Y_{1}-Y_{0}-\lambda\right)\right)}
$$

In the canonical lattice basis, these generating functions are structure constants for the product

$$
Y_{0} Y_{1}:=\sum_{Y_{2} \in K\left(L_{k_{2}+k_{1}}\right)_{0}} A_{Y_{1}-Y_{0}}^{\left[k_{1} k_{2} /\left(k_{2}+k_{1}\right)\right]} Y_{2} .
$$

This expression is compatible with Seidel's mirror map in the following sense. Let $\widetilde{T}:=\left(\Lambda_{0} \oplus \Lambda^{\prime *}\right) \otimes \mathbb{R} /\left(\Lambda_{0} \oplus \Lambda^{\prime *}\right)$ be the dual torus, mirror to $T$. If $M, B$ and $N$ are as in Section 2, then $\widetilde{T}$ has complex structure

$$
\widetilde{J}:=\left(\begin{array}{cc}
1 & 0 \\
B & 1
\end{array}\right)\left(\begin{array}{cc}
0 & -M^{-1} \\
M & 0
\end{array}\right)\left(\begin{array}{cc}
1 & 0 \\
-B & 1
\end{array}\right)
$$

while the mirror of the endomorphism $J_{f}$ is the polarization

$$
E_{f}:=\left(\begin{array}{cc}
0 & -N^{\mathrm{T}} \\
N & 0
\end{array}\right)
$$

which is compatible with $\widetilde{J}$ by the symmetry of $N^{\mathrm{T}} M$. Therefore, $E_{f}$ is the first Chern class of some line bundle (of zero characteristics) $\widetilde{L}$. Assume that $\widetilde{L}$ is ample. We denote by $H_{f}$ the Hermitian form associated to $E_{f}$ 
and by $S_{f}$ the $\mathbb{C}$-linear extension of $H_{f}$ restricted to $\left(\Lambda^{\prime *} \otimes \mathbb{R}\right) \times\left(\Lambda^{* *} \otimes \mathbb{R}\right)$. Moreover, let

Then, for all $x, y \in L_{0}$,

$$
\widetilde{J}_{b}:=\left(\begin{array}{cc}
0 & -B^{-1} \\
B & 0
\end{array}\right)
$$

$$
\left(H_{f}-S_{f}\right)(x, y):=E_{f}(\widetilde{J} x, y)-i E_{f}\left(\widetilde{J}_{b} x, y\right)=(\omega-i b)(x, f(y)) .
$$

This identification allows us to compare the Fukaya product with the classical product of theta functions. We follow the treatment of [5]. A canonical basis for $H^{0}(\widetilde{T}, \widetilde{L})$ is given by the following theta functions:

$$
\begin{aligned}
\theta_{c}^{\widetilde{L}}(v):= & \mathrm{e}^{\left((\pi / 2) S_{f}(v, v)-(\pi / 2)\left(H_{f}-S_{f}\right)(c+2 v, c)\right)} \\
& \times \sum_{\lambda \in \Lambda\left(L_{k}\right)_{0}} \mathrm{e}^{\pi\left(H_{f}-S_{f}\right)(c+v, \lambda)-(\pi / 2)\left(H_{f}-S_{f}\right)(\lambda, \lambda)},
\end{aligned}
$$

where $v \in\left(\Lambda_{0} \oplus \Lambda^{* *}\right) \otimes \mathbb{R}$ and $c \in K\left(L_{0}\right)_{0}$. Moreover, the product

$$
\widetilde{m}: H^{0}(\widetilde{T}, \widetilde{L}) \otimes H^{0}(\widetilde{T}, \widetilde{L}) \longrightarrow H^{0}\left(\widetilde{T}, \widetilde{L}^{2}\right)
$$

can be expressed on this basis as

$$
\widetilde{m}\left(\theta_{c_{1}}^{\widetilde{L}} \otimes \theta_{c_{2}}^{\widetilde{L}}\right)=\sum_{c_{3} \in K\left(L_{2}\right)_{0}} \theta_{c_{2}-c_{1}}^{\widetilde{L}^{2}}(0) \theta_{c_{3}}^{\widetilde{L}^{2}}
$$

Since the elements of the canonical bases for $\operatorname{Hom}_{D F u k(T)}$ and $\operatorname{Hom}_{\mathcal{D}(\widetilde{T})}$ are both labeled by elements in $K(L)_{0}$, it is natural to put them in correspondence $Y_{c} \leftrightarrow \theta_{c}$. In this way, we identify the generators of the ring $\mathcal{R}$ with the generators of the homogenous coordinate ring of the projective embedding of the mirror complex torus $\widetilde{T}$. Given a relation $\sum C^{c_{1}, \ldots, c_{n}} Y_{c_{1}} \cdots Y_{c_{n}}=0$ in $\mathcal{R}$, we can reduce it to a linear one using the product (4.1). Replacing $Y_{c} \leftrightarrow \theta_{c}$ everywhere yields another valid linear relation, as both sets of generators are linearly independent over $\mathbb{C}$. Finally, using the product (4.2) and the identity

$$
\theta_{c}^{\widetilde{L}^{2 k}}(0)=\sum_{\lambda} \mathrm{e}^{-k \pi\left(H_{f}-S_{f}\right)(c-\lambda, c-\lambda)}=A_{c}^{[k]},
$$

we can work backwards and obtain the relation $\sum C^{c_{1}, \ldots, c_{n}} \theta_{c_{1}}, \ldots, \theta_{c_{n}}=$ 0 . Clearly, this process can be reversed and we conclude that $\mathcal{R}$ and the homogeneous coordinate ring of the embedded mirror are isomorphic.

At least in principle, we can apply the point of view of [14] and present, using only the knowledge of the Fukaya category of $T$, the mirror torus $\widetilde{T}$ as an explicit complete intersection in some projective space, uniquely specified by the Lagrangian $L_{0}$ and the symplectomorphism $\rho$. For example, a set of simple relations can be recovered as follows. Let $m_{k}: \mathcal{R}^{\otimes k} \rightarrow \mathcal{R}$ be the $k$-fold multiplication. Then for any $z \in \mathcal{R}$ of degree $k$ and for any pair $x_{1} \otimes \cdots \otimes x_{k}, y_{1} \otimes \cdots \otimes y_{k}$ such that $x_{i}, y_{i} \in \mathcal{R}$ all of degree 1 and 
$m_{k}\left(x_{1} \otimes \cdots \otimes x_{k}\right)=\alpha_{x} z, \quad m_{k}\left(y_{1} \otimes \cdots \otimes y_{k}\right)=\alpha_{y} z$, then $\alpha_{y}\left(x_{1}, \ldots, x_{k}\right)=$ $\alpha_{x}\left(y_{1}, \ldots, y_{k}\right)$ is clearly a relation in $\mathcal{R}$. In the language of theta-functions, for $k=2,3$, these relations are classically known as Riemann's theta relations and cubic theta relations, respectively. The linear map $f$ can be chosen so that the above relations generate any other relation in $\mathcal{R}$.

\section{Dependence on $\rho$}

In this section, we investigate some examples and study how the mirror family depends on the choice of symplectomorphism around the large complex structure point.

\subsection{Twisted homogeneous coordinate rings}

So far, we assumed the symplectomorphism $\rho_{f}$ to be strictly linear. Relaxing this condition and allowing affine symplectomorphisms, one can reconstruct twisted homogeneous rings as well. As an example, we now show that the noncommutative projective plane can be reconstructed from a symplectic two-torus together with the symplectomorphism $\rho(x, y)=(x+b, y+3 x)$ for any $b \neq \mathbb{Z}$. If $b \in \mathbb{Z}$ we recover the situation of [14] from which we adapt the notation as follows. Let $T=\mathbb{R}^{2} / \mathbb{Z}^{2}$ be the torus with coordinates $x, y$ and symplectic form $\tau d x \wedge d y$. In the universal cover, we define three Lagrangians $L_{0}:=\{y=0\}, \quad L_{1}:=\{y=3(x-b)\}$ and $L_{2}:=\{y=6(x-$ $3 b / 2)\}$ for some $b \in \mathbb{R}$. Passing to the quotient, $L_{0} \cap L_{1}=\left\{X_{i}:=(i / 3+\right.$ $b, 0) \mid i=0,1,2\}, L_{0} \cap L_{2}=\left\{Y_{j}:=(j / 6+3 b / 2,0) \mid j=0, \ldots, 5\right\}$ and $L_{1} \cap L_{2}$ $=\{(k / 3+2 b, k+3 b) \mid k=0,1,2\}=\rho\left\{X_{0}, X_{1}, X_{2}\right\}$. For general $b \in \mathbb{R}$, the product formula of [14] becomes

$$
X_{i} X_{j}=\sum_{k=0}^{1} A_{i-j+3 k}(b) Y_{i+j+3 k},
$$

where we have put

$$
A_{k}(b):=\sum_{n} \mathrm{e}^{i \pi 6 \tau(n+k / 6+b / 2)^{2}}=\theta\left[\frac{k}{6}+\frac{b}{2}, 0\right](6 \tau, 0) .
$$

If $b \in \mathbb{Z}$, commutativity is ensured by the relation $A_{k}(b)=A_{6-k}(-b)$. For $b \notin \mathbb{Z}$, we get the relations

$$
\begin{aligned}
& p X_{2}^{2}+q X_{0} X_{1}+r X_{1} X_{0}=0 \\
& p X_{1}^{2}+q X_{2} X_{0}+r X_{0} X_{2}=0 \\
& p X_{0}^{2}+q X_{1} X_{2}+r X_{2} X_{1}=0
\end{aligned}
$$


where

$$
p:=A_{1} A_{2}-A_{4} A_{5}, \quad q:=A_{3} A_{4}-A_{0} A_{1}, \quad r:=A_{0} A_{5}-A_{3} A_{2}
$$

and $b$ dependence is understood. The noncommutative algebra, which is the quotient of the noncommutative (associative) homogeneous polynomial ring $\mathbb{C}\left\{X_{0}, X_{1}, X_{2}\right\}$ by the above relations, is known as the Sklyanin algebra $\operatorname{Skl}_{3}(p, q, r)$. If $b \in \mathbb{Z}, \operatorname{Skl}_{3}(p, q, r)$ simply reduces to the ring of homogeneous polynomials in three variables and so when $b \notin \mathbb{Z}$, it makes sense to interpret it as the homogeneous coordinate ring of a noncommutative projective plane. Numerical checks confirm that elliptic curve $E$ with modular parameter $\tau$ (i.e., the mirror of $T$ ) has the equation

$$
Q_{p q r}:=X_{0}^{3}+X_{1}^{3}+X_{2}^{3}-\frac{p^{3}+q^{3}+r^{3}}{p q r} X_{0} X_{1} X_{2}=0
$$

On the other hand, $Q_{p q r}$ generates the center of $\operatorname{Skl}_{3}(p, q, r)$ and (as shown in $[3,4,6])$

$$
\frac{\mathrm{Skl}_{3}(p, q, r)}{Q_{p q r}} \cong \bigoplus_{n \geq 1} H^{0}\left(E, L \otimes\left(t_{b}\right)^{*} L \otimes \cdots \otimes\left(\left(t_{b}\right)^{n-1}\right)^{*} L\right)
$$

where $L$ is the degree 3 line bundle on $E$ which defines the projective embedding such that $E=\operatorname{Proj}\left(C\left[X_{0}, X_{1}, X_{2}\right] / Q_{p q r}\right)$ and $t_{b} \in \operatorname{Aut}(E)$ is the translation by $b$. Moreover, the category of coherent sheaves on $E$ embeds into the category of graded modules (up to torsion) over $\operatorname{Skl}_{3}(p, q, r)$ which, in the language of [4], justifies the assertion that the mirror of $T$ sits as a commutative curve into a noncommutative projective space.

\subsection{Quasihomogeneous coordinate rings}

Consider the two-torus of [14] again, but with $\rho(x, y)=(x, y+x)$ rather than the choice $(x, y+3 x)$ which led to cubic curves in the Hesse family. The appearance of quasihomogeneous coordinate rings will be quite natural. To do the calculation, we return to the philosophy of [14], where the mirror map was found without any prior knowledge of it. The relations will be shown to agree with Section 4.

We have $L_{k}=\{y=k x\}$. Let $X$ be the lattice point $0 \in L_{0} \cap L_{1}, Y_{0}$ and $Y_{1}$ the points $Y_{k}=(k / 2,0) \in L_{0} \cap L_{2}$, and $Z_{0}, Z_{1}, Z_{2}$ the points 
$Z_{k}=(k / 3,0) \in L_{0} \cap L_{3}$. Compute $X^{2}=A_{0}^{[2]} Y_{0}+A_{1}^{[2]} Y_{1}$, where we define

$$
A_{j}^{[k]}=\theta\left[\frac{j}{k}, 0\right](k \tau, 0), \quad j \in \mathbb{Z} / k \mathbb{Z} .
$$

We put $Y=Y_{0}$ and $Z=Z_{1}$. Then relations will necessarily be quasihomogeneous with respect to the grading $|X|=1,|Y|=2,|Z|=3$. Also note that using the (commutative) Fukaya product, we can express $Z_{k}$ in terms of $X^{3}$, $X Y$ and $Z{ }^{1}$ Similarly, the results of [14] allow us to readily express the six points $W_{k}=(k / 6,0) \in L_{0} \cap L_{6}$ in terms of products $Z_{i} Z_{j}$. We only require $W_{0}=a_{1} Z_{0}^{2}-a_{3} Z_{1} Z_{2}$ and $W_{2}+W_{4}=a_{1}\left(Z_{1}^{2}+Z_{2}^{2}\right)-a_{3}\left(Z_{0} Z_{1}+Z_{0} Z_{2}\right)$, where $a_{k}=A_{k}^{[6]} /\left(A_{0}^{[6]} A_{1}^{[6]}-A_{2}^{[6]} A_{3}^{[6]}\right)$. Writing the $W_{k}$ in terms of bilinears in $X^{3}, X Y$ and $Z$ gives six of the seven quasihomogeneous monomials of degree six in $X, Y$, and $Z$. The remaining one is $Y^{3}$, which we calculate in terms of the $W_{k}$ as

$$
Y^{3}=\left(A_{0}^{[4]} A_{0}^{[12]}+A_{2}^{[4]} A_{6}^{[12]}\right) W_{0}+\left(A_{0}^{[4]} A_{4}^{[12]}+A_{2}^{[4]} A_{2}^{[12]}\right)\left(W_{2}+W_{4}\right) .
$$

This gives a single relation in degree six.

Now given a polynominial equation as

$$
F \equiv Y^{3}-\left(p_{0} Z^{2}+p_{1} X Y Z+p_{2} X^{2} Y^{2}+p_{3} X^{3} Z+p_{4} X^{4} Y+p_{6} X^{6}\right)=0,
$$

it is a simple matter to make "linear" changes of variables (such as $Z \rightarrow$ $\left.Z+\left(1 / 2 p_{0}\right)\left(p_{1} X Y+p_{3} X^{3}\right)\right)$ to put the equation in a form with $p_{0}=1 / 4$, $p_{1}=p_{2}=p_{3}=0$. Then working in an affine patch with coordinates $y=$ $Z / X^{3}, x=Y / X^{2}$, the equation has the Weierstrass form $y^{2}=4 x^{3}-g_{2} x-$ $g_{3},{ }^{2}$ from which one reads $j(\tau)=1728 g_{2}^{3} /\left(g_{2}^{3}-27 g_{3}^{2}\right)$. Plugging in the power series as outlined above and in the footnotes, we find (up to the first few dozen coefficients checked by computer) the usual integer $q$-series expansion for $j$, with $q=\mathrm{e}^{2 \pi i \tau}$, i.e., the elliptic curve has modular parameter $\tau$, and the usual mirror map is established.

\footnotetext{
${ }^{1}$ Define

$$
\mu=\frac{A_{0}^{[2]} A_{0}^{[6]}+A_{1}^{[2]} A_{3}^{[6]}}{A_{0}^{[2]} A_{2}^{[6]}+A_{1}^{[2]} A_{1}^{[6]}}, \quad \nu=\frac{A_{0}^{[6]}}{A_{2}^{[6]}}
$$

Then

$$
\begin{aligned}
& Z_{0}=\frac{1}{\left(A_{0}^{[2]} A_{2}^{[6]}+A_{1}^{[2]} A_{1}^{[6]}\right)(\mu-\nu)} X^{3}-\frac{1}{A_{2}^{[6]}(\mu-\nu)} X Y, \quad Z_{1}=Z, \\
& \text { and } Z_{2}=-\frac{\nu}{\left(A_{0}^{[2]} A_{2}^{[6]}+A_{1}^{[2]} A_{1}^{[6]}\right)(\mu-\nu)} X^{3}-\frac{\mu}{A_{2}^{[6]}(\mu-\nu)} X Y-Z .
\end{aligned}
$$

${ }^{2}$ Define $t_{2}=p_{2}-p_{1}^{2} /\left(4 p_{0}\right), \quad t_{4}=p_{4}-p_{1} p_{3} /\left(2 p_{0}\right), \quad t_{6}=p_{6}-p_{3}^{2} /\left(4 p_{0}\right) . \quad$ Then $g_{2}^{3}=$ $\left(2 / p_{0}\right)^{2}\left[t_{4}+t_{2}^{2} / 3\right]^{3}$ and $g_{3}^{2}=\left(1 / p_{0}\right)^{2}\left[t_{6}+t_{4} t_{2} / 3+2 t_{2}^{3} / 27\right]^{2}$.
} 
The ring $\mathcal{R}=\mathbb{C}[X, Y, Z] / F$ describes the mirror elliptic curve $F=0$ inside weighted projective space $\mathbb{P}^{2}(1,2,3)$ (see [13]). To compare with the quadratic relations of Section 3, one considers the seven monomials

$$
V_{0}, \ldots, V_{6}=X^{6}, X^{4} Y, X^{3} Z, X^{2} Y^{2}, X Y Z, Y^{3}, Z^{2}
$$

as homogeneous projective coordinates on $\mathbb{P}^{6}$. The nine quadratic relations such as $V_{0} V_{4}=V_{1} V_{2}$ describe the (image of the) Veronese embedding $\mathbb{P}^{2}(1,2,3) \hookrightarrow \mathbb{P}^{6}$. In this description, the relation $F=0$ is linear in the $V_{k}$, i.e., the projective line bundle has a unique section - the mirror of $X \in L_{0} \cap L_{1}$.

\subsection{Kummer varieties}

Seidel's method applies to quotients of abelian varieties as well. By definition, a Kummer surface is the quotient of an abelian variety with respect to the involution which reverses the orientation of the lattice. It is a singular surface with 16 singularities, and if it can be embedded as a hypersurface in $\mathbb{P}^{3}$ it has equation $[8]$

$$
\begin{aligned}
& A\left(X_{0}^{4}+X_{1}^{4}+X_{2}^{4}+X_{3}^{4}\right)+B\left(X_{0}^{2} X_{1}^{2}+X_{2}^{2} X_{3}^{2}\right)+C\left(X_{0}^{2} X_{2}^{2}+X_{1}^{2} X_{3}^{2}\right) \\
& \quad+D\left(X_{0}^{2} X_{3}^{2}+X_{1}^{2} X_{2}^{2}\right)+2 E X_{0} X_{1} X_{2} X_{3}=0
\end{aligned}
$$

We claim that we can reconstruct such a Kummer surface from a real fourtorus $T:=\mathbb{R}^{4} / \mathbb{Z}^{4}$ endowed with complex symplectic form

$$
\omega=\tau_{1} d x_{1} \wedge d y_{1}+\tau_{2} d x_{2} \wedge d y_{2}+\tau_{3}\left(d x_{1} \wedge d y_{2}+d x_{2} \wedge d y_{1}\right),
$$

the standard involution $\iota\left(x_{1}, y_{1}, x_{2}, y_{2}\right)=-\left(x_{1}, y_{1}, x_{2}, y_{2}\right)$ and the symplectomorphism $\rho\left(x_{1}, y_{1}, x_{2}, y_{2}\right)=\left(x_{1}, y_{1}+2 x_{1}, x_{2}, y_{2}+2 x_{2}\right)$. On $K:=T / \iota$,

$$
\begin{gathered}
\left|L_{0} \cap \rho L_{0}\right|=\left(\begin{array}{l}
4 \\
1
\end{array}\right) \quad\left|L_{0} \cap \rho^{2} L_{0}\right|=\left(\begin{array}{l}
5 \\
2
\end{array}\right) \quad\left|L_{0} \cap \rho^{3} L_{0}\right|=\left(\begin{array}{l}
6 \\
3
\end{array}\right) \\
\left|L_{0} \cap \rho^{4} L_{0}\right|=\left(\begin{array}{l}
7 \\
4
\end{array}\right)-1
\end{gathered}
$$

so that, generically, among the homogeneous polynomials in $X_{0}, \ldots, X_{3} \in$ $L_{0} \cap \rho L_{0}$, we expect one relation in degree 4 to define the ring $\mathcal{R}$ with no relations in degree 2 or 3 . The computation of $\mathcal{R}$ for general Kummer varieties will appear elsewhere [2].

To give an idea of the computation, here we illustrate the degenerate case where $\tau:=\tau_{1}=\tau_{2}$ and $\tau_{3}=0$. In particular, the matrices $M, B$ and $N$ (see Section 2) are simultaneously diagonalizable, a situation that is mirror to the case of an abelian surface polarized by the square of a reducible principal 
polarization. Therefore we expect (e.g., from [8], Proposition 4.23) a map onto a quadric in $\mathbb{P}^{3}$. In the universal cover, consider the Fukaya products

$$
Y_{(a, b)}^{[k]} Y_{(c, d)}^{[k]}:=\sum_{i, j \in \mathbb{Z} / 2 \mathbb{Z} \times \mathbb{Z} / 2 \mathbb{Z}} A_{c-a+2 k i}^{[2 k]} A_{d-b+2 k j}^{[2 k]} Y_{(a+c+2 k i, b+d+2 k j)}^{[2 k]}
$$

where

$$
Y_{(a, b)}^{[k]}:=\left(\frac{a}{2 k}, 0, \frac{b}{2 k}, 0\right) \in L_{0} \cap \rho^{k} L_{0}
$$

In particular, defining

$$
X_{0}:=Y_{(0,0)}^{[1]}, \quad X_{1}:=Y_{(1,0)}^{[1]}, \quad X_{2}:=Y_{(0,1)}^{[1]}, \quad X_{3}:=Y_{(1,1)}^{[1]},
$$

we have

$$
X_{0} X_{3}=A_{1}^{[4]} A_{1}^{[4]}\left(Y_{(1,1)}^{[2]}+Y_{(1,3)}^{[2]}+Y_{(3,1)}^{[2]}+Y_{(3,3)}^{[2]}\right)=X_{1} X_{2}
$$

The last equation defines the image of a Veronese embedding $\mathbb{P}^{1} \times \mathbb{P}^{1} \hookrightarrow \mathbb{P}^{3}$. On the other hand, $\rho$ can be restricted to $\rho_{i}\left(x_{i}, y_{i}\right)=\left(x_{i}, y_{i}+2 x_{i}\right), i=1,2$. The corresponding Seidel map $\left(\mathbb{R}^{2} / \mathbb{Z}^{2}, \omega_{i}=\tau d x_{i} \wedge d y_{i}\right) \rightarrow \mathbb{P}^{1}$ can be used to find the equation of the mirror elliptic curve in weighted projective space $\mathbb{P}(1,1,2)$, as in the last section. The mirror map defined by $\rho$ is seen to be the composition of the Veronese embedding and the Cartesian product of the maps arising from $\rho_{1}$ and $\rho_{2}$.

\section{Acknowledgments}

We would like to thank Paul Seidel for sharing his ideas. We are grateful to The Fields Institute for partial support and for hosting us while parts of this project were completed. The work of E.Z. was supported in part by a Clay Senior Scholars fellowship and by NSF grant DMS-0405859. Any opinions, findings and conclusions or recommendations expressed in this material are those of the authors and do not necessarily reflect the views of the National Science Foundation (NSF).

\section{References}

[1] M. Abouzaid, Homogeneous coordinate rings and mirror symmetry for toric varieties, math.SG/0511644.

[2] M. Aldi, Twisted homogeneous coordinate rings of abelian surfaces via mirror symmetry, math.SG/0605648.

[3] D. Auroux, L. Katzarkov and D. Orlov, Mirror symmetry for weighted projective planes and their noncommutative deformations, math.AG/0404281. 
[4] M. Artin, J. Tate and M. Van den Bergh, Some algebras associated to automorphisms of elliptic curves, The Grothendieck Festschrift, vol 1., Birkhauser, Boston, (1990), 33-85.

[5] Ch. Birkenhake and H. Lange, Cubic theta relations, J. Reine Angew. Math. 407 (1990), 167-177.

[6] A. Bondal and A. Polishchuk, Homological properties of associative algebras: the method of helices (translation), Russ. Acad. Sci. Izv. Math. 42 (1994), 216-260.

[7] K. Fukaya, Mirror symmetry of abelian varieties and multi theta functions, preprint available at http://www.math.kyoto-u.ac.jp/ fukaya/fukaya.html.

[8] M. R. Gonzalez-Dorrego, Configurations and geometry of Kummer surfaces in $\mathbb{P}^{3}$, Mem. AMS 107 (1994), 512.

[9] K. Hori, S. Katz, A. Klemm, R. Pandharidpande, R. Thomas, C. Vafa, R. Vakil and E. Zaslow, Mirror symmetry, Clay Math. Monogr., 1, AMS, Providence, RI/CMI, Cambridge, MA, 2003.

[10] M. Kontsevich and Y. Soibelman, Homological mirror symmetry and torus fibrations, Symplectic Geometry and Mirror Symmetry, World Scientific, 2001; math.SG/0011041.

[11] A. Polishchuk, Abelian varieties, theta functions and the Fourier transform, Cambridge University Press, Cambridge, UK, 2003.

[12] A. Polishchuk and E. Zaslow, Categorical mirror symmetry: the elliptic curve, Adv. Theor. Math. Phys. 2 (1998), 443-470; math.AG/9801119.

[13] M. Reid, Graded rings and varieties in weighted projective space, www.maths.warwick.ac.uk/ miles/surf/more/grad.pdf.

[14] E. Zaslow, Seidel's mirror map for the torus, Adv. Theor. Math. Phys. 6 (2005); math.SG/0506359. 